\title{
Las marcas de posesión en wayunaiki*
}

\author{
RUDECINDO RAMÍREZ GONZÁLEZ **
}

Recepción: 28 de mayo de 2021

Aprobación: 10 de septiembre de 2021

Forma de citar este artículo: Ramírez, R. (2021). Las marcas de posesión en wayunaiki. Cuadernos de Lingüística Hispánica, (38), e12893

(di) https://10.19053/0121053X.n38.2021.12893

* Artículo de investigación. En el presente artículo se exponen resultados parciales de la investigación "Categorías gramaticales relacionadas con el nombre en wayunaiki", tesis doctoral en Lingüística de la Universidad de Antioquia, 2018..

** Doctor en Lingüística de la Universidad de Antioquia, Medellín, Colombia; profesor titular de la Universidad de La Guajira, Colombia. Grupo de investigación A’in, motor de vida; rramirez@uniguajira.edu.co (D) https:// orcid.org/0000-0001-7115-0573 


\section{Resumen}

El objetivo de este estudio es analizar e interpretar las marcas morfológicas que remiten la posesión en el contexto gramatical del nombre; además, demostrar cómo se construye una estructura posesiva nominal en esta lengua. El análisis de los datos recolectados en terreno se hizo desde la perspectiva de la lingüística descriptiva (Himmelmann, 1998; Chelliah \& De Reuse, 2011), que incluye la recolección de datos de primera fuente, la detección de patrones regulares que subyacen al funcionamiento de los aspectos gramaticales estudiados y su examen a partir de categorías y terminología que gozan de difusión general en trabajos descriptivos.

En wayunaiki se distinguen marcas de posesión: las marcas de posesión inalienable y las marcas de posesión alienables. A la categoría de inalienable pertenecen los nombres que remiten a las partes del cuerpo humano, los términos de parentesco, las partes del cuerpo de los animales, las partes de las plantas, algunos nombres inanimados que remiten a cosas estrechamente relacionadas con los humanos. Morfológicamente, el wayunaiki trata diferente a los nombres inalienables y a los alienables.

Palabras clave: wayunaiki, posesión, inalienable, alienable, marcas morfológicas, nombre, pronominal.

\section{Marks of Possession in Wayuunaiki}

\section{Abstract}

The objective of this paper is to analyze and interpret the morphological marks that refer to possession in the grammatical context of the name; likewise, demonstrate how a nominal possessive structure is built in this language. The analysis of the data collected in the field was done from the perspective of descriptive linguistics (Himmelmann, 1998; Chelliah \& De Reuse, 2011), which includes the collection of first-source data, the detection of regular patterns that underlie the functioning of the grammatical aspects studied and their study based on categories and terminology that are widely disseminated in descriptive works.

In Wayuunaiki, position marks are distinguished: the inalienable possession marks and the alienable possession marks. To the category of inalienable belong the names that refer to the parts of the human body, the terms of kinship, the parts of the body of animals, the parts of plants, some inanimate names that refer to things closely related to humans. Morphologically, Wayuunaiki treats inalienable and alienable names differently.

Keywords: Wayuunaiki, possession, inalienable, alienable, morphological marks, noun, pronominal. 


\section{Les marques de possession dans le wayunaiki}

\section{Résumé}

L'objectif de cette étude est d'analyser et d'interpréter les marqueurs morphologiques qui font référence à la possession dans le contexte grammatical du nom, et de démontrer comment une structure possessive nominale est construite dans cette langue. L'analyse des données recueillies in situ a été faite dans la perspective de la linguistique descriptive (Himmelmann, 1998 ; Chelliah \& De Reuse, 2011), qui comprend la collecte de données de source primaire, la détection de modeles réguliers sous-jacent le fonctionnement des aspects grammaticaux étudiés, et leur examen sur la base de catégories et d'une terminologie largement utilisées dans les travaux descriptifs.

Dans le wayunaiki, une distinction est faite entre les marques de possession : les marques de possession inaliénable et les marques de possession aliénable. Dans la catégorie des inaliénables, il appartient les noms se référant aux parties du corps humain, les termes de parenté, les parties du corps des animaux, les parties des plantes, certains noms inanimés se référant à des choses étroitement liées aux humains. Morphologiquement, le wayunaiki traite différemment les noms inaliénables et aliénables.

Mots clés: wayunaiki, possession, inaliénable, aliénable, marques morphologiques, nom, pronom, pronominal.

\section{Marcas de possessão em wayuunaiki}

\section{Resumo}

0 objetivo deste estudo é analisar e interpretar as marcas morfológicas que se referem à possessão no contexto gramatical do nome; também, demonstre como uma estrutura possessiva nominal é construída nesta linguagem. A análise dos dados coletados em campo foi feita na perspectiva da lingüística descritiva (Himmelmann, 1998; Chelliah \& De Reuse, 2011), que inclui a coleta de dados de primeira fonte, a detecção de padrões regulares que fundamentam o funcionamento de os aspectos gramaticais estudados e seu exame com base em categorias e terminologias amplamente difundidas em obras descritivas.

Em wayunaiki, as marcas de posse são distinguidas: as marcas de posse inalienável e as marcas de posse alienável. À categoria de inalienáveis pertencem os nomes que se referem às partes do corpo humano, os termos de parentesco, as partes do corpo dos animais, as partes das plantas, alguns nomes inanimados que se referem a coisas intimamente relacionadas aos humanos. Morfologicamente, o wayunaiki trata nomes inalienáveis e alienáveis de maneira diferente.

Palavras-chave: wayunaiki, possessão, inalienável, alienável, marcas morfológicas, nome, pronominal 


\section{Introducción}

Este artículo ofrece una visión descriptiva de la expresión de la posesión, como una categoría gramatical asociada con el nombre en la lengua wayunaiki ${ }^{1}$. En el marco de una investigación sobre la morfología del nombre y categorías asociadas en esta lengua, a continuación se presenta un análisis de rigor de la expresión de la posesión, que, adyacente con las de género, número y marcas preposicionales, instituyen las más importantes de categorías gramaticales que se suelen expresar en el nombre y sus modificadores en las lenguas naturales.

El wayunaiki ${ }^{2}$ es una lengua indígena hablada por el grupo étnico wayuu en el departamento de La Guajira, la zona más septentrional de Colombia (Instituto Geográfico Agustín Codazzi [IGAC], 1986), y en el estado Zulia en la parte noroccidental de la República Bolivariana de Venezuela, donde forma territorio fronterizo colombovenezolano en la zona caribeña de ambas naciones. Esta lengua es la más hablada de la familia arawak en Colombia ${ }^{3}$,por una población ${ }^{4}$ de entre doscientos mil y trescientos mil hablantes.

Este artículo se estructura del siguiente modo, después de esta introducción, se presentan las consideraciones teóricas básicas que sustentan el estudio de datos lingüísticos de la lengua wayuu. Luego hay una síntesis de los resultados de trabajos anteriores sobre el tema y seguidamente, una descripción de la metodología empleada en la investigación. A continuación, se expone un análisis de las marcas de posesión desde una perspectiva gramatical de prefijos y sufijos presentes en el radical del nombre que codifica información sobre posesión. Por último, se enuncian las conclusiones de este trabajo.

\section{Consideraciones teóricas: "posesión inherente"/“posesión establecida"}

Buena parte de los trabajos sobre posesión son en general descritos en términos de las categorías alienable e inalienable. Aunque estas categorías han sido tratadas a menudo en investigaciones lingüísticas, no dejan de presentar algunos problemas en su definición, debido específicamente a dos interpretaciones preponderantes con respecto a la inalienabilidad: una, en términos estructurales, para referir a un conjunto de sustantivos ligados que no pueden manifestarse sin un marcador posesivo; y otra, en términos semánticos, para aludir a los casos en que no se puede concebir el poseído sin hacer referencia al poseedor (Regúnaga, 2009, p. 42).

1 En la literatura sobre esta lengua se usan diferentes términos para denominarla: guajiro, wayuunaiki, wayuu. En el presente artículo se empleará el término wayunaiki.

2 Es una palabra compuesta por: wayu.anüiki que traduce persona.lengua, lengua de los wayuu

3 Según datos suministrado por Aikhenvald (1999), el wayunaiki pertenece a la familia arawak.

4 Según datos consultados recientemente, la población de hablantes del wayunaiki es de 394683 (DANE, 2018). 
Seiler (1983, p. 5) acude con ciertas reservas a los términos "alienable" e "inalienable" por considerarlos engañosos; en cambio, prefiere utilizar "posesión inherente" y "posesión establecida". Según este autor, prototípicamente, la POSESIÓN inherente es morfológicamente no marcada y altamente gramaticalizada. Semánticamente designa una relación íntima entre EL POSEEDOR y POSSESSUM, tal como se puede encontrar en lo que tradicionalmente se ha denominado "sustantivos inalienables”. Además, señala que los casos más prominentes de POSESIÓN inherente ocurren en construcciones que implican partes del cuerpo y términos de parientes como POSSESSUM, y pronombres personales o nombres propios como POSEEDOR.

Como ya se dijo, la noción a la que remite el posesivo no es fácil de delimitar, ya que se trata de diferentes tipos de relaciones muy variadas y complejas, como lo expresa Creissels (2006):

Il est banal que l'interprétation la plus spontanée de la construction génitivale ou des possessifs ne soit pas 'possessive' au sens ordinaire de ce terme : ma mère, mon ami, mon patron, mon médecin, ma rue (la rue où j'habite), mon train (le train que je vais prendre), ma banque (la banque où j'ai ouvert un compte), mon école (l'école que je fréquente), mon assiette (l'assiette qui m'a été attribuée, lors d'un repas au restaurant), ma photo (la photo qui me représente), etc. Plutôt qu'autour de la notion de possession au sens que ce terme peut avoir dans le langage ordinaire, le sémantisme du génitif et des possessifs s'organise autour de la notion de participation d'une entité (communément désignée comme possédé ) à la sphère personnelle d'un individu (communément désigné comme possesseur ) : la sphère personnelle d'un individu est l'ensemble des entités avec lesquelles on peut considérer que cet individu entretient une relation plus ou moins privilégiée, quelle que soit la nature précise de cette relation. (p. 143)

Un estudio importante sobre la posesión en varias lenguas es el de Aikhenvald (2012), quien afirma:

The linguistic expression of 'possession' within a noun phrase can be viewed as one of the realizations of a broader concept of association or relationship between two nouns (...). Or there may be one or more dedicated possessive noun phrase types which cover the core meanings associated with Possessive relationship (...). (p. 2)

Además, en su obra analiza categorías gramaticales posesivas y no posesivas, y los significados recurrentes de "núcleos" realizados a través de construcciones posesivas. Según su estudio, en muchas lenguas del mundo, una frase posesiva con nombres se usa para expresar partes del cuerpo humano, términos de parentesco, de propiedad y asociación en general. La autora (2012) señala que los términos de parentesco, las partes del cuerpo se pueden expresar de una manera y las de propiedad de otra. Esto quiere decir que, en determinada lengua, las partes del cuerpo y los términos de parentesco se poseen directamente a través de sufijos que se adhieren fijamente al nombre. Cuando el poseedor léxico no está presente, este puede 
expresarse con afijos posesivos personales, casi siempre prefijos (Dixon \& Aikhenvald, 1999).

Para la expresión formal de la posesión (en su sentido amplio y no literal) existen en las lenguas naturales muchas estrategias: la yuxtaposición de nombres (en un cierto orden fijo, que puede variar de lengua a lengua), la afijación al nombre poseído de marcas personales que indican el posesor (ya sean sufijos o prefijos), la afijación de marcas de posesivo directamente al nombre para indicar que este último es el posesor, el uso de preposiciones que indican posesión, la combinación de varias de estas categorías, entre otras (Bickel \& Nichols, 2013). Esto quiere decir que las lenguas codifican la posesión de diversas formas y que pueden tener más de una para expresar dicho contenido. Así, muchas lenguas tienen sistemas binarios de clasificación posesiva, en tanto unas pocas tienen sistemas más complejos. Por el contrario, hay lenguas que generan oposiciones entre dos formas de marcas posesivas condicionadas por las propiedades del elemento que opera como poseído en la construcción.

\section{Antecedentes del estudio de la posesión en wayunaiki}

El autor venezolano Álvarez (1996) describe las principales construcciones posesivas del guajiro [wayunaiki]. Identificó morfemas prefijados que son empleados para indicar poseedor y sufijos posesivos: -se, -ni, -ya. Además, detectó verbos posesivos y privativos con prefijos $k v$ - y $m v$-. El análisis de su trabajo se centró en examinar la distinción morfológica entre nombres absolutos y relativos. Llama la atención que Álvarez (1996) determine el morfema posesivo -in como -ni.

Los autores Álvarez y Urdaneta (2005) relacionan los nombres alienables como absolutos y los inalienables como relativos. Resaltan que los inalienables son intrínseca y obligatoriamente poseídos con un prefijo pronominal. Uno de los problemas encontrados por los autores es que la distribución de los sufijos de posesión no es predecible. A algunos nombres se les agrega el sufijo de posesión -se, a otros se les agrega el sufijo -in, -se es más frecuente y productivo

El resultado de nuestro trabajo de investigación sobre la posesión en wayunaiki se aproxima a los resultados de los trabajos de Álvarez (1996) y Álvarez y Urdaneta (2005), básicamente en los siguientes aspectos: en wayunaiki se distingue una oposición de nombres inalienables y alienables. Los inalienables (posesión inherente) necesitan prefijos de persona para indicar la posesión nominal. Los alienables requieren tanto de prefijos de persona como de sufijos de posesión -in, -se. El grupo de nombres inalienables incluye partes del cuerpo humano, términos de parentesco, algunos objetos de pertenencia, las partes de las plantas. Los alienables se dividen en forma no poseída y forma poseída. Los nombres alienables poseídos llevan marcas prefijadas de persona poseedor y sufijos de posesión. Los no poseídos se refieren a fenómenos de la naturaleza, los nombres de personas y de lugares mitológicos y son libres o no ligados, los cuales no pueden ser poseídos. El prefijo $a$ - 
de los grupos de nombres inalienables es denominado en estos trabajos como marca de indefinido.

\section{Metodología de la recolección de datos}

La presente investigación se realizó con base en datos recogidos en terreno con hablantes bilingües (wayunaiki-español) de la comunidad de Nazareth y Media Luna en la región de la Alta Guajira, entre 2014 y 2017. Los cuatro informantes tenían entre 29 y 53 años, dos pertenecen a la comunidad de Nazareth y dos a la de Medialuna. Se escogió esta región porque sus hablantes en general se consideran más competentes en wayunaiki que en las demás regiones de la península.

El corpus está conformado por diversos tipos de muestra recolectadas: grabación de narraciones y diálogos espontáneos, así como cuestionarios diseñados para la elicitación del fenómeno específico estudiado, la posesión. En el caso de los cuestionarios, se les pidió a los hablantes la traducción oral (y en algunos casos escrita) del español al wayunaiki, de estructuras que podrían contener expresiones de posesión, es decir, nombres de diversos tipos con sus categorías asociadas. Uno de los cuestionarios contenía sintagmas nominales posesivos, y el otro cuestionario, oraciones transitivas e intransitivas con sintagmas nominales de posesión.

\section{Análisis de las marcas de posesión en wayunaiki}

La posesión es una categoría gramatical que va marcada en la morfología del nombre en wayunaiki. La posesión se marca directamente en el nombre, al igual que la definitud, el género y el número. En wayunaiki se distinguen dos clases de nombres: los que son semánticamente inalienables y llevan obligatoriamente una marca posesiva, y los alienables, que pueden ser poseídos o no y que no llevan marca posesiva por obligación.

\section{La posesión inherente (tradicionalmente llamada nombres inalienables)}

Los nombres inherentes se definen por el hecho de marcar morfológicamente una relación de posesión de manera obligatoria. A la posesión inherente en wayunaiki pertenecen los nombres que remiten a las partes del cuerpo humano, los términos de parentesco, las partes del cuerpo de los animales, las partes de las plantas, algunos nombres inanimados que aluden a cosas estrechamente relacionadas con los humanos. En los términos de parentesco y en las partes del cuerpo se adhieren directamente las marcas de posesión. Estos nombres aparecen siempre precedidos por un pronombre personal posesivo, que ocurre de manera prefijada (ligada) y que señala la referencia al posesor. 
(1)

$\begin{array}{lll}\text { Persona } & \text { Posesión } & \text { Glosa } \\ \text { 1SG } & \text { ta-tüna } & \text { 'mi brazo' } \\ \text { 2SG } & \text { pü-tüna } & \text { 'tu brazo' } \\ \text { 3SG.M } & \text { nü-tüna } & \text { 'su brazo' (de él) } \\ \text { 3SG.F } & \text { jü-tüna } & \text { 'su brazo' (de ella) } \\ \text { 1PL } & \text { wa-tüna } & \text { 'nuestro brazo' } \\ \text { 2PL } & \text { jü-tüna } & \text { 'su brazo' (de ustedes) } \\ \text { 3PL.M } & \text { na-tüna } & \text { 'su brazo' (de ellos) }\end{array}$

(2)

$\begin{array}{lll}\text { Persona } & \text { Posesión } & \text { Glosa } \\ \text { 1sG } & \text { te-ki } & \text { 'mi cabeza' } \\ \text { 2SG } & \text { pi-ki } & \text { 'tu cabeza' } \\ \text { 3sG.m } & \text { ni-ki } & \text { 'su cabeza' (de él) } \\ \text { 3SG.F } & \text { ji-ki } & \text { 'su cabeza' (de ella) } \\ \text { 1PL } & \text { we-ki } & \text { 'nuestra cabeza' } \\ \text { 2PL } & \text { ji-ki } & \text { 'su cabeza' (de ustedes) } \\ \text { 3PL.m } & \text { ne-ki } & \text { 'su cabeza' (de ellos) }\end{array}$

Es importante que señalar que hay variación morfofonológica en el caso de que el nombre en cuestión comience por vocal, como por ejemplo eki ("cabeza"), caso en el que o bien se elide la vocal final del pronombre, como en $t(a)$-eki, o la vocal inicial del nombre como en $p i$-(e) $k i$, además de que se usa la variante $p i$-, $n i$-, $j i$ - en vez de $p \ddot{u}, n \ddot{u}$-, jü-, como se ve en la serie presentada en (2). Estos fenómenos morfofonológicos deberán ser estudiados a fondo en trabajos futuros.

Los siguientes son ejemplos con el término de parentesco -oushi ('abuela'):

(3)

$\begin{array}{lll}\text { Persona } & \text { Posesión } & \text { Glosa } \\ 1 \text { ss } & \text { to-ushi } & \text { 'mi abuela' } \\ 2 S G & \text { po-ushi } & \text { 'tu abuela' }\end{array}$




$\begin{array}{lll}\text { 3SG.M } & \text { no-ushi } & \text { 'su abuela' (de él) } \\ \text { 3SG.F } & \text { so-ushi } & \text { 'su abuela' (de ella) } \\ \text { 1PL } & \text { wo-ushi } & \text { 'nuestra abuela' } \\ \text { 2PL } & \text { jo-ushi } & \text { 'su abuela' (de ustedes) } \\ \text { 3PL.M } & \text { no-ushi } & \text { 'su abuela' (de ellos) }\end{array}$

Como se observa en los ejemplos anteriores, los términos de parentescos y las partes del cuerpo aparecen con prefijos posesivos pronominales obligatorios que marcan la posesión. Las partes del cuerpo son miembros que están íntimamente unidos a la persona, no se conciben como aislados ni desprendidos, son algo que le pertenece a uno o se posee. Los parientes establecen relaciones sociales muy cercanas en el grupo étnico, hay expresión de dependencia. Es decir, son entidades dependientes, en el sentido de que es inherentemente definido en términos de otro objeto del cual es una parte. En términos semánticos, los nombres de las partes del cuerpo se refieren a miembros que siempre están unidos al cuerpo, nunca están aislados.

Algunas entidades inanimadas que aluden a espacios y queguardan una relación muy estrecha con las personas, especialmente la vivienda, como en (6), forman parte también de los nombres inherentes; por ejemplo, la puerta de la casa es "ojo" de la vivienda", la fuente de agua es "lágrima de tierra", entre otros. Frecuentemente, para denominar las partes de las viviendas y de los objetos domésticos se usan únicamente los pronominales posesivo singulares y plurales de la 3sg.m nü- ni- o 3sg.f jü$j i$ - sü-, 3pl.m na-, también para las partes del cuerpo de los animales como en (7) y las partes de las plantas como en (13). Todos los pronominales posesivos se usan para la posesión de las partes del cuerpo y términos de parentesco humano como en los ejemplos (1), (2) y (3).

(4) nü-wuira wottikoso

3SG.M-lágrima wottikoso

'lágrimas de Wottikoso'

(5) ji-wuira mma

3SG.F-lágrimas tierra

'lágrimas de la tierra' (= fuente/manantial)

$5 \quad$ Pero en la actualidad se tienden a usar préstamos del español: 'puerta' = puetta. 
(6) ji-o'u kusinapia-ka-t

3SG.F-ojo cocina-DEF-F.SG

el ojo (la puerta) de la cocina'

(7) a nü-si ama-ka-i

3SG.M-RABO CABALLO-DEF-M

'el rabo del caballo'

b. jü-wala ama-ka-t

3SG.M-pelo caballo-DEF-F

'el pelo de la yegua'

Los pronombres personales prefijados, que aparecen de manera obligatoria en los nombres inherentes, son:

Tabla 1. Pronombres ligados al nombre para expresar el posesor

\begin{tabular}{|l|l|}
\hline \multicolumn{2}{|l|}{ Pronombres ligados al nombre para expresar el poseedor } \\
\hline 1SG & $t a$ - 'mi' \\
\hline 2SG & $p \ddot{u}$ - / pi- 'tuyo', 'suyo' \\
\hline 3SG.M & $n \ddot{u}$ - / ni- 'su' (de él) \\
\hline 3SG.F & $j u ̈$ - / ji- 'su' (de ella) \\
\hline 1PL & $w a$ - 'nuestro' \\
\hline 2PL & $j \ddot{u}$ - / ji-'suyo' (de ustedes) \\
\hline 3PL.M & $n a$-'su' (de ellos) \\
\hline 3.PL.F & $j \ddot{u}$ - / ji- -rua 'su' (de ellas) \\
\hline
\end{tabular}

Estos pronombres 0 índices de persona prefijados no se pueden omitir en los nombres posesivo-inherentes (si se omiten, la oración resulta agramatical), como se ve en los ejemplos siguientes:

(8)

ta-salir-ü-in ta-o'u

$\left(*_{0}^{\prime} \mathrm{u}\right)$

1SG-lastimar-PAS-OD 1SG-0jo

'me lastimé mi ojo'

(9)

ant-ü-shi nü-siipü Peetut

(*siipü) 
llegar-PAS-M.SG 3SG-sobrino Pedro

'llegó el sobrino de Pedro'

(10) jewe-sü jü-chon ${ }^{6}$ tü jamüche'-ka-t (*chon)

maduro-F.SG 3SG.F-fruto DEF.F.SG tuna-DEF-F.SG

'el fruto de la tuna está maduro'

(11)

wa-epia tü (*epia)

\section{PL-vivienda DEF.F.SG}

'esta es nuestra vivienda'

En el ejemplo (8), el elemento posesor ta- en ta-salir-ü-in es el sujeto de la oración con verbo transitivo, y la entidad en función de objeto es el elemento poseído (ta-o’u). En (9) el orden varía: el elemento poseído es el sujeto de la oración con verbo intransitivo y el objeto es el posesor.

Estos nombres inherentes opcionalmente llevan marca de definitud, género, plural y otros afijos modificadores, pero esto no es obligatorio y depende del sentido que el hablante quiera darle. Por ejemplo, si en el ejemplo (12) el hablante quiere precisar que se trata del cuñado específico del que están hablando (un cuñado definido) y no de cualquiera de sus cuñados, probablemente agregará las marcas de definitud, género y número.

(12) ootolir-aa-shi ni-nanchi-ka-i mma-lu'u

acostar-PRES-M 3SG.M-cuñado-DEF-M.SG suelo-INES

'su cuñado se acuesta en el suelo'

En los ejemplos siguientes, el hablante no consideró necesario - en (13) precisar a cuál de sus hombros se refiere, y en (14) no es necesario marcar como definida la única cabeza a la que puede referirse:

no-o'ot-ü-in nü-nut-pa'

3SG.M-subir-PAS-OD 3SG.M-hombro-SIT

'lo montó en su hombro'

nü-eit-ü-in ni-eki-püna

6 La palabra para "hijo" (chon) se usa igual para "fruto" y proviene del sufijo diminutivo -chon. Esta relación léxica entre "hijo" y "fruto", y entre estos dos términos y el diminutivo es usual en las lenguas de la familia arawak (Grand 2017, comunicación personal). 


\section{SG.M-disparar-PAS-OD 3SG.M-cabeza-SUB}

'él le disparó por debajo de su cabeza'

Cuando lo poseído es inherente, la estructura predominante de estas construcciones es la siguiente:

$$
\text { PRONposesor - Nposeído + Nposesor }
$$

En las construcciones posesivas con nombres inherentes puede aparecer el posesor realizado léxicamente como en $(4,5,6,7,9$, ), o este puede no aparecer, como en $(11,13$ y 14). En ambos casos, la presencia del pronombre prefijado que indica el posesor debe aparecer obligatoriamente en el nombre que remite a lo poseído. Para dar cuenta de esta opcionalidad de realización léxica del posesor, la siguiente estructura es más adecuada:

$$
\text { PRONposesor - Nposeído + (Nposesor) }
$$

La posesión inherente está menos marcada morfológicamente que la posesión alienable, es decir, se expresa con menos marcas, como se verá en el apartado siguiente.

\section{La posesión establecida (tradicionalmente conocida como nombres alienables)}

La posesión establecida, en un sentido semántico, refiere a entidades que pueden ser o pueden no ser poseídas. A diferencia de una parte del cuerpo, como un brazo, que siempre va a pertenecerle a una y a la misma persona (y es, por lo tanto, posesión inherente), un objeto como una olla, por ejemplo, hoy le puede pertenecer a alguien, pero si mañana es vendida, regalada o botada a la basura, le pertenecerá a otra persona o no tendrá dueño, es decir, es posesión establecida.

Se entiende entonces que, en el contexto de lo inherente, se trata de una posesión permanente, perenne como las partes del cuerpo humano, como por ejemplo en (15a) y (16a). En cambio, en el caso de los nombres de posesión establecida, que son poseídos solo ocasionalmente, una entidad se percibe como de pertenencia o de propiedad de alguien, que es el dueño de los objetos obtenidos y usados para beneficio personal, como en los ejemplos (15b) y (16b). En wayunaiki se consideran entidades poseíbles ocasionalmente algunos objetos o entidades como las herramientas, el dinero, los utensilios de cocina, la vestimenta, los animales domésticos, etc.

(15) a. ni-o'u Juan
3SG.M-ojo Juan
'el ojo de Juan'
b. ni-o'u-se Juan 
.M-OJo-POS JuAN

'las gafas de Juan'

(16) a. jü-awala Karmen

3SG.F-pelo Carmen

'el pelo de Carmen'

b. jü-peinia-in Karmen

3SG.F-peinilla-POS Carmen

'la peinilla de Carmen'

Morfológicamente, el wayunaiki trata de manera diferente a los nombres de posesión inherente y a los de posesión establecida. Mientras que los de posesión inherente obligatoriamente deben llevar marcas posesivas (el pronombre prefijado que remite al posesor), los de posesión establecida pueden llevar marcas posesivas 0 no, según si se desea expresar una relación de posesión, como en (17a), o no, como en (17b).

(17) a. jeke-tü nü-anua-in Jüse

nuevo-F.SG 3SG.M-canoa-POS José

'la canoa de José es nueva'

b. pü-süsalaja ta-müin wanee anua

2SG-regalar 1SG-DAT INDEF canoa

'regálame una canoa'

Es decir, "ojo" nunca aparecerá sin un prefijo de posesor, mientras que "canoa" puede aparecer con el prefijo o no, según si se desea señalar a un posesor.

En los siguientes ejemplos, así como en los ejemplos (15b), (16b) y (17a), se observa que las entidades poseídas, cuando son posesión establecidas, llevan no solo la marca morfológica que señala el posesor, es decir, el pronombre prefijado, sino que también llevan un sufijo -in o -se:

$$
\begin{aligned}
& \text { awat-aa-sü jüi-püliiku-se Maria } \\
& \text { trabajar-PRES-F.SG 3SG.F-burra-POS María } \\
& \text { 'la burra de María está corriendo' } \\
& \text { ni-eira-in wanee wayu epieyu }
\end{aligned}
$$


3SG.M-canto-POS INDEF wayu epieyu

'el canto de un wayu epieyu'

(20) akumaj-ü-shi taya wanee piichi jü-lu'u ta-ma-in

construir-PAS-M.SG 1SG INDEF casa 3SG.F-INES 1SG-tierra-POS

'construí una casa en mi territorio'

(21) o'otoj-ee-chi joolu' taya ni-a'u ta-ama-in

montar-FUT-M.SG ahora 1SG 3SG.M-SUP 1SG-caballo-POS

'yo voy a montar ahora en mi caballo'

(22) ayapaj-aa-shi pi-kamisa-in

coser-PRES-F.SG 2SG-camisa-POS

'tú coses tu camisa'

(23) ojutt-ü-sü nü-luma-in Kushemata

derrumbar-PAS-F.SG 3SG.M-enrramada-POS Kushemata

'se derrumbó la enramada de Kushemata'

(24) jala-sü nü-anua-in nia ya

dónde-F 3SG.M-canoa-POS 3SG.M INTERR

‘¿dónde está la canoa de él?'

(25) jala-sü anua jeket-ka-t ya

dónde-F canoa nueva-def-f interr

'dónde está la canoa nueva'

Estos sufijos -in y -se marcan lo poseído, y no se encuentran en los nombres inherentes. Son dos, entonces, las diferencias morfológicas que se constatan entre nombres alienables e inalienables en wayunaiki: 1 . La obligatoriedad de la aparición de una marca de posesor, en el caso de los nombres de posesión inherentes. 2. La presencia de prefijos pronominales y el uso necesario de sufijos que marcan el nombre de posesión establecida como poseído, mientras que la aparición de estos sufijos en los nombres de posesión inherentes es agramatical. Hay casos en que los nombres 
de posesión establecida ${ }^{7}$ pueden aparecer sin las marcas de prefijo pronominal y los sufijos de posesión como en (17b) y (25), o sea, no son nombres poseídos.

En la Tabla 2 se muestra una variedad de nombres que designan instrumentos, medios de transporte acuático, objetos de uso personal y nombres abstractos y que llevan -in como marca de posesivo.

Tabla 2. Lista de algunos nombres poseídos marcados con el morfema -in

\begin{tabular}{|l|l|l|}
\hline Alienables & Poseídos & Glosa \\
\hline polú (f) & -polu-in & 'hacha' \\
\hline anua (f) & -anua-in & 'cayuco' \\
\hline iita (f) & -iita-in & 'totumo' \\
\hline utia (f) & -utia-in & 'aguja' \\
\hline rouya (f) & -rouya-in & 'espejo' \\
\hline si'raj (f) & -si'ra-in & 'faja' \\
\hline kamiisa (f) & -kamiisa-in & 'camisa' \\
\hline lapü (f) & -lapü-in & 'sueño' \\
\hline
\end{tabular}

En la Tabla 3 encontramos ejemplos de nombres que corresponden a seres sexuados que llevan marcas de género. En este grupo de nombres se encuentra tanto el morfema -in como el morfema -se antepuesto a las marcas de definido y de género. Como se dijo, tanto los nombres de posesión inherente como los establecidos pueden ir seguidos de sufijos determinantes del nombre, que marcan el género, el número y la definitud.

Tabla 3. Nombres de seres sexuados poseídos que llevan marcas de género y definitud

\begin{tabular}{|l|l|l|}
\hline Nombres alienables & Nombres poseídos & Glosa \\
\hline ama & -ama-in-ka-i & 'el caballo' \\
\hline ama & -ama-in-ka-t & 'la yegua' \\
\hline annet & -annet-se-ka-i & 'el ovejo' \\
\hline annet & -annet-se-ka-t & 'la oveja hembra' \\
\hline püliiku & -püliiku-se-ka-t & 'la burra' \\
\hline püliiku & -püliiku-se-ka-i & 'el burro' \\
\hline
\end{tabular}

7 Etiquetas enajenables opcionalmente posesivas o no posesivas (Nichols \& Bickel, 2013). 
En la Tabla 4 encontramos una lista de los nombres alienables que van marcados con el morfema -se. Como se puede ver, hay nombres femeninos y masculinos. La lista incluye nombres de seres animados e inanimados ${ }^{8}$.

Tabla 4. Lista de nombres poseídos marcados con -se

\begin{tabular}{|l|l|l|}
\hline Nombres alienables & Nombres poseídos & Glosa \\
\hline kaliina (f) & -kaliina-se & 'gallina' \\
\hline kotoleere (m) & -kotoleere-se & 'gallo' \\
\hline yuui (f) & -yuui-se & 'tabaco' \\
\hline kusinapia (f) & -kusinapia-se & 'cocina' \\
\hline womü (f) & -womü-se & 'sombrero' \\
\hline waireiya (f) & -waireiya-se & 'sandalia' \\
\hline aseyuu (f) & -aseyuu-se & 'espíritu' \\
\hline riwoot (m) & -riwot-se & 'revolver' \\
\hline sittija (m) & -sittija-se & 'anillo' \\
\hline neerü (f) & -nee-se & 'dinero' \\
\hline chajaruuta (f) & -chajaruuta-se & 'machete' \\
\hline rüi (m) & -rüi-se & 'cuchillo' \\
\hline susu (f) & -susia-se & 'mochila' \\
\hline yüüja (f) & -yuuja-se & 'huerta' \\
\hline
\end{tabular}

Es claro que los morfemas -in y -se son sufijos que indican lo poseído, ya que ocurren siempre en construcciones posesivas con nombres de posesión establecida. Sin embargo, no hemos podido determinar en qué contexto se utiliza cada uno de estos sufijos. Según los datos que aparecen en las tablas y en el resto de nuestro corpus, en general predomina el sufijo -se. Pero ni los criterios fonológicos (terminación de la raíz) ni criterios suprasegmentales (acentuación) ni el número de sílabas ni una hipotética armonía vocálica ni el género inherente o marcado del nombre poseído ni el rasgo semántico de animado/no animado permiten proponer una regularidad en el comportamiento de los sufijos -in y -se. Parece ser que la asignación de una u otra forma fuera impredecible 0 arbitraria, aunque se constata la tendencia general de que los nombres poseídos de género inherente masculino llevan -se y no -in. Estudios futuros cuantitativos y con un corpus más amplio podrán tal vez arrojar luz sobre este punto.

8 Hay algunos nombres a los que es difícil asignarles un género específico, ya que los hablantes les asignan a veces el género masculino y a veces el femenino, como en el caso de e’jentü (f)/(m) "vehículo" y wunu'u (m)/(f) "palo". 
Hay que anotar que las construcciones que llamamos aquí posesivas deben entenderse en un sentido amplio, es decir, en el sentido de que no expresan únicamente una relación de "posesión" en sentido estricto, sino que remiten, como en la mayoría de las lenguas naturales, a una relación más amplia (por lo que también se podrían denominar construcciones genitivas). de posesión:

En el siguiente ejemplo se ve que la relación expresada no es estrictamente la

$$
\begin{aligned}
& \text { ni-ap-aa-in nü-kolektiwa-se Kushemata } \\
& \text { 3SG.M-coger-PRES-OD 3SG.M-colectivo-GEN Kushemata } \\
& \text { 'Kushemata coge su (bus) colectivo' }
\end{aligned}
$$

Kushemata no posee el bus colectivo, sino que es el que suele usar o el que le corresponde en esa ocasión.

En cuanto a la distinción entre los nombres de posesión inherentes y los establecidos, el wayunaiki se asemeja tipológicamente a otras lenguas de la familia arawak, por ejemplo, al lokono y al añú (De Carvalho, 2016), aunque no se hará aquí una comparación detallada de las lenguas en cuestión, ya que el presente trabajo no pretende ser tipológico, sino describir y analizar el sistema de una sola lengua.

A continuación, se resumen (Tabla 5) las estructuras de las construcciones posesivas inherentes y establecidas (recuérdese que la marca de posesión pronominal posesor en los inherentes y en los establecido es obligatoria).

Tabla 5. Construcciones posesivas con nombres alienables e inalienables

\begin{tabular}{|l|l|}
\hline Inalienables & Alienables \\
\hline PRON $_{\text {POSESOR }}-\mathrm{N}_{\text {POSEÍDO }}\left(\mathrm{N}_{\text {POSESOR }}\right)$ & PRON $_{\text {POSESOR }}-\mathrm{N}_{\text {POSEÍDO- }}$ POS $_{\text {POSEÍ́O }}\left(\mathrm{N}_{\text {POSESOR }}\right)$ \\
\hline
\end{tabular}

\section{Conclusiones}

La oposición entre nombres inherente y establecidos es muy evidente en wayunaiki. Los inherentes (partes del cuerpo, los términos de parentesco, relaciones espaciales, las partes de las plantas, etc.) aparecen siempre con prefijos posesivos obligatorios y son categorías netamente de poseídos. Los establecidos, según si se desea expresar una relación de posesión, llevan marca posesiva. Además, los nombres establecidos llevan no solo la marca morfológica que señala el posesor, es decir, un pronombre prefijado, sino que también llevan un sufijo -in 0 -se que marcan la posesión de las entidades poseídas (cosas u objetos de propiedad). La posesión está típicamente marcada en nombres poseídos. En la frase posesiva el orden más extendido de palabra es poseído-poseedor. 
El nombre poseído lleva un prefijo personal que concuerda con el nombre poseedor, además, lleva adicionalmente un sufijo posesivo, si se trata de un nombre de posesión establecido, pero no lo lleva si se trata de un nombre inherente (partes de cuerpo y parentesco). Cuando el poseedor léxico no está presente, el poseedor puede expresarse con afijos posesivos personales, casi siempre prefijos.

\section{Abreviaturas}

$1 \mathrm{PL}=1 \cdot{ }^{\mathrm{a}}$ persona plural

$1 \mathrm{SG}=1 .^{\mathrm{a}}$ persona singular

$2 \mathrm{SG}=2 \cdot{ }^{\mathrm{a}}$ persona singular

$2 \mathrm{PL}=2 \cdot{ }^{\mathrm{a}}$ persona plural

3PL.M $=3 \cdot{ }^{\mathrm{a}}$ persona plural masculino

$3 S G . F=3{ }^{a}$ persona singular femenino

3SG.M $=3 \cdot^{\mathrm{a}}$ persona singular masculino

DAT $=$ dativo

$\mathrm{DEF}=$ definido

$\mathrm{F}=$ femenino

FUT $=$ futuro

$\mathrm{GEN}=$ genitivo

INDEF $=$ indefinido

INES $=$ inesivo

INTERR $=$ interrogativo

$\mathrm{M}=$ masculino

$\mathrm{N}=$ nombre

$\mathrm{OD}=$ objeto directo

PAS = pasado

$\mathrm{PL}=$ plural

PRON $=$ pronombre

$\mathrm{POS}=$ posesivo 
PRES $=$ presente

$\mathrm{SG}=$ singular

SIT $=$ sitiativo

$\mathrm{SUB}=$ subersivo

SUP $=$ supersivo

\section{Referencias}

Aikhenvald, A. Y. (1999). The Arawak Language Family. In R.M.W. Dixon \& A. Y. Aikhenvald (Eds.). The Amazonian Languages, (pp. 65-106). Cambridge University Press.

Aikhenvald, A. Y. (2012). Possession and Ownership: A Cross Linguistic Perspective. Oxford University Press.

Álvarez, J. (1996). Construcciones posesivas en guajiro. Opción, (19), 21-44.

Álvarez, J. \& Urdaneta, J. (2005). Haplología en los sufijos de posesión en guajiro/ wayuuniki. Lingua Americana, (16), 119-148.

Bickel, B. \& Nichols J. (2013) Obligatory Possessive Inflection. In M.S. Dryer, \& M. Haspelmath (eds.) The World Atlas of Language Structures Online. Max Planck Institute for Evolutionary Anthropology.

Chelliah, S. L. \& Willem J. de Reuse (2011). Handbook of Descriptive Linguistic Fieldwork. Springer.

Creissels, D. (2006). Syntaxe générale. En: Une introduction typologique. Vols. 1 y 2. Lavoisier.

De Carvalho, F. (2016). The Diachrony of Person-Number Marking in the Lokonowayuunaiki Subgroup of the Arawak Family. Language Sciences, (55), 1-15.

DANE. (2018). Censo Nacional de Población 2018; resultados preliminares. DANE.

Dixon, R. M. W., \& Aikhenvald, A. Y. (Eds.) (1999). The Amazonian Languages. Cambridge Language Surveys. Cambridge University Press.

Himmelmann, N. P. (1998). Documentary and Descriptive Linguistics. Linguistics (36), 161-195.

Instituto Geográfico Agustín Codazzi -IGAC-. (1986) La Guajira: aspectos geográficos. IGAC. 
Nichols, J. \& Bickel, B. (2013). Possessive Classification. In M. S. Dryer \& M. Haspelmath (eds.), The World Atlas of Languaje Estructure Online. Max Planck Institute for Evolutionary Antropology.

Nichols,J. \& Bickel, B. (2005). Possessive classification and obligatory possessive. In: Haspelmath, M.; Dryer, M.; Gil, D.; Comrie, B. The world atlas of language structures. Oxford: Oxford University Press, 242-245.

Regúnaga, M.A. (2009). La relación entre posesión y género en tehuelche. Liames 9, 41-60.

Seiler, H. (1983). Possession as An Operational Dimension of Language. Language Universals Series, 2. Günter Narr Verlag. 